\title{
LA HERMENÉUTICA GADAMERIANA COMO SÍNTESIS ENTRE EL ENFOQUE CUANTITATIVO Y CUALITATIVO EN LA INVESTIGACIÓN SOCIAL
}

\author{
GADAMERIAN HERMENEUTICS AS A SYNTHESIS BETWEEN THE QUANTITATIVE \\ AND QUALITATIVE APPROACH IN THE SOCIAL RESEARCH
}

\author{
Esteban Rodríguez Moya* \\ Universidad de Talca - Universidad Católica del Maule, \\ Talca - Chile.
}

Recibido enero de 2019/Received January, 2019

Aceptado marzo de 2019/Accepted March, 2019

\begin{abstract}
RESUMEN
En la actualidad sigue estando presente la disputa entre los partidarios de los enfoques cuantitativo y cualitativo al interior de las ciencias sociales. Sin embargo, al examinar el problema el acento siempre se ha puesto en las aristas metodológicas de la discusión, dejando de lado los elementos epistemológicos y filosóficos que puede haber de fondo. De esta manera, se entiende que una solución más significativa de la cuestión debe necesariamente venir dada a través de una propuesta filosófica, la cual, junto con plantear un fundamento para la realidad o su conocimiento, pueda vincularse también con las características más relevantes de cada enfoque. En este contexto, el presente escrito busca plantear el pensamiento hermenéutico de Hans-Georg Gadamer como posible solución a las diferencias mencionadas, pues sus alcances universales, su acentuación en la interpretación y su noción circular de la comprensión humana la proveen de elementos que la asocian claramente con la filosofía, la epistemología, la investigación cualitativa y la investigación cuantitativa.
\end{abstract}

Palabras Clave: Método científico, Enfoque cuantitativo, Enfoque cualitativo, Hermenéutica gadameriana, filosofía.

\begin{abstract}
The dispute between the supporters of quantitative and qualitative approaches within the social sciences continues to be present. However, when examining the problem the accent has always been placed on the methodological edges of the discussion, leaving aside the epistemological and philosophical elements that may be in the background. In this way, it is understood that a more significant solution to the issue must necessarily come through a philosophical proposal, which, together with raising a foundation for reality or its knowledge, can also be linked to the most relevant characteristics of each approach. In this context, the present paper seeks to propose the hermeneutic of Hans-Georg Gadamer as a possible solution to the mentioned differences, because its universal scope, its accentuation in the interpretation and its circular notion of human understanding provide it with elements that associate it clearly with the philosophy, the epistemology, the qualitative research and the quantitative research.
\end{abstract}

Key Words: Scientific method, Quantitative approach, Qualitative approach, Gadamerian hermeneutics, Philosophy.

\section{INTRODUCCIÓN}

En la actualidad aún existen diferencias entre los partidarios de las metodologías cuantitativas y los partidarios de las metodologías cualitativas a nivel de la investigación científica social. Tal como plantean Johnson y Onwuegbuzie (2004), muchas veces estas disputas se ven alimentadas

* Autor correspondiente / Corresponding author: erodriguez631@ gmail.com 
por convicciones tales como el positivismo, que entiende que los fenómenos sociales pueden ser estudiados como los fenómenos naturales ${ }^{1}$, o, al otro lado de la confrontación, la idea de que no se puede separar el objeto de conocimiento y el sujeto que conoce, pues se aprecia a la dimensión subjetiva como única base para percibir la realidad (Guba, 1990, como se citó en Johnson \& Onwuegbuzie, 2004). Así, aparece lo que Scribano (2000) denomina como "prejuicio teórico" en torno a las diferencias existentes entre estrategias cuantitativas y cualitativas, las que de esta forma pasan a conformar paradigmas rivales e inconmensurables. Por otra parte, si entendemos la epistemología como la reflexión filosófica en torno a los fundamentos del conocimiento científico, es fácil constatar que en el fondo de la confrontación se encuentran perspectivas epistemológico-filosóficas de difícil conciliación. De esta manera, surge la interrogante por propuestas que provengan de la filosofía y que a la vez aúnen dentro de sí características que sean transversales a ambas perspectivas dentro de la investigación en ciencias sociales.

En relación a lo anterior, el presente texto se presenta como una revisión teórica de carácter especulativo, en la cual se pretende ofrecer un principio heurístico y filosófico que ayude a establecer un mismo fundamento entre ambos paradigmas metodológicos. Para orientarnos en el cumplimiento de este objetivo, se tendrá como supuesto de investigación que la hermenéutica de Hans-Georg Gadamer representa un punto de unión entre los enfoques cuantitativo y cualitativo dentro de la investigación social, pues abarca simultáneamente aspectos filosóficos, interpretativos y metodológico-positivistas. De este modo, primero nos abocaremos a exponer algunas características del método científico, repasando la disputa y vínculos metodológicos posibles entre cuantitativistas y cualitativistas. En un segundo momento revisaremos la propuesta hermenéutica gadameriana, específicamente la noción del comprender que le es propia. Finalmente, se propondrá el planteamiento hermenéutico citado como síntesis de los dos enfoques presentes en la actividad científica social.

\section{UNA SÍNTESIS DEL MÉTODO CIENTÍFICO}

Las dudas que pueden existir en torno al método científico y su cohesión a través de la
Historia, sumiéndolo en aquello que podríamos llamar de acuerdo a Feyerabend (1986) como anarquismo epistemológico, o los cambios de paradigma que son propios a las revoluciones científicas y que pueden afectar tanto a su fundamento epistemológico como a sus prácticas metodológicas (Kuhn, 2013), no evitan que podamos definir y explicar el método científico propiamente tal. Sobre todo, si al momento de hacerlo se le abarca desde una perspectiva general sin ahondar en sus problemas particulares. En este sentido, al observar el funcionamiento de la ciencia a nivel macro y en su configuración actual ${ }^{2}$, tenemos que esta se enmarca dentro de un conocimiento sistemático de la realidad que se caracteriza por tener un contenido, un campo de estudio y una forma de actuación concretas (Sierra, 1987). Ahora bien, respecto específicamente de su contenido y campo de estudios, encontramos que estos se forman por ideas (teorías) y hechos (fenómenos) respectivamente, lo cual no diferencia a las ciencias de otras formas de conocimiento que también pueden extraer ideas a partir de hechos. Por este motivo, lo que en realidad distingue a la ciencia como forma de conocimiento es su forma de actuación, es decir el método científico. Éste puede definirse someramente como una forma de acción general que se sigue para lograr conocimiento científico (Sierra, 1987) o como una secuencia ordenada de operaciones en pos de obtener conocimiento (Bunge \& Ardila, 1988). De este modo, dentro de la forma de acción, científica podemos encontrar según Sierra (1987) tres pasos centrales ${ }^{3}:$ 1) Formular un problema con base en la observación y la teoría previa. 2) Anticipar una solución al problema a través de una hipótesis. 3) Corroborar con los hechos mismos ${ }^{4}$ la solución anticipada o hipótesis por medio de la observación, la experimentación, la clasificación y el análisis. Gracias a este método la ciencia adquiere ciertas características que de algún modo delimitan lo que podemos y no podemos considerar como tal. Asimismo, por su marcado acento en la apelación a los hechos para confirmar las hipótesis que surgen a partir de un cuerpo teórico, la ciencia es: teórica, problemáticohipotética, empírica, deductivo-inductiva, crítica, circular, analítico-sintética, selectiva e imaginativa (Sierra, 1987). Al momento de hacer una síntesis de todas estas características, o destacar una de las más importantes, claramente deberíamos aludir a la circularidad. La circularidad metodológica 
de la ciencia resume todo el recorrido de sus pasos al momento de generar conocimiento. Sin duda, parte desde la teoría, una red nomológica compuesta de principios científicos, para dar respuesta a un problema en específico. Dichas respuestas tienen un carácter provisorio en forma de modelos e hipótesis mientras no sean confirmadas por medio de experimentos. Sin embargo, si la respuesta provisoria es validada a través de la observación (y la experimentación) implica un regreso a la teoría misma, pues la modifica o la complementa en mayor o menor grado. A su vez, esto conlleva que la ciencia tenga un movimiento de ida con carácter deductivo, donde se infieren posibles respuestas frente a un problema (debiendo ser corroboradas con los acontecimientos). Pero también, esto comporta un movimiento de regreso con carácter inductivo, donde se extrae información a partir de los datos del experimento, los cuales al ser interpretados confirman o no las hipótesis propuestas, produciendo también impacto en el cuerpo teórico del cual emergieron. Sierra (1987) resume este movimiento a partir de la Figura 1, donde la circularidad del proceso de corroboración permite generar o enriquecer las ideas científicas.

Figura 1.

Proceso de corroboración y circularidad

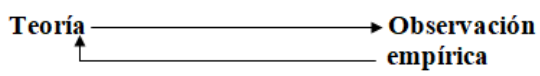

Existe una cohesión metodológica aparente a través de la estructura circular del método científico, es correcto afirmar aparente porque si bien es cierto que esta circularidad está presente en gran parte de la investigación científica, también es claro que su apartado referente a la observación y comprobación de las ideas supuestas a través de los hechos trae problemas a la hora de aplicar el método científico a la realidad social. Es sabido que las ciencias sociales aplican este método a la realidad social con el fin de responder a problemas de diversa índole en dicho campo (Sierra, 1987). Estos problemas se vinculan en su mayoría a las conductas o comportamientos sociales, los cuales muchas veces dependen de elementos que no son fácilmente observables y cuantificables como, por ejemplo, los valores morales que no tienen una realidad objetiva externa (Sierra, 1987). Todo esto ha llevado a que muchos investigadores se adscriban a una forma cualitativa de trabajar, donde lo importante es la interpretación y la conceptualización (cualidad) de la realidad sociocultural ${ }^{5}$ por sobre la cuantificación y el experimento. Así, los partidarios del análisis cualitativo se han enfrascado en una lucha con los partidarios de una forma de investigación social que interpreta más convencionalmente el método científico, estos últimos son los llamados cuantitativistas ${ }^{6}$. Así pues, para entender que esta disputa tiene más una profundidad epistemológica que metodológica es necesario examinar con mayor detención ambas perspectivas de investigación social citadas, así como también sus posibles puntos comunes y de ruptura.

\section{PERSPECTIVAS CUANTITATIVAS Y CUALITATIVAS EN CIENCIAS SOCIALES. PUNTOS DE UNIÓN Y RUPTURA}

Repasando brevemente lo planteado en la introducción, tenemos que tanto el enfoque teórico cuantitativo como el cualitativo pueden ser vinculados a presupuestos o visiones filosóficas determinadas. En este sentido, el primero se vincularía al ya mencionado positivismo ${ }^{7}$, pues coincide con una visión materialista de la realidad que conlleva una unidad del método científico (Moyano, 1999). Mientras que por su parte la segunda se instala dentro de una perspectiva hermenéutica, culturalista e historicista, debido a que posee un marcado realce en las significaciones compartidas e históricamente construidas (Moyano, 1999). Por otro lado, es importante recordar también que la disputa protagonizada por ambas perspectivas se resume en la distancia que existe entre concebir a las cosas o a las ideas como fundamento de la realidad. Así pues, y como se pude intuir, la pugna goza de largos siglos, específicamente desde la antigüedad griega con Platón y Aristóteles (Moyano, 1999).

Se pueden postular variados intentos de combinación o unión entre los enfoques cuantitativo y cualitativo. Por ejemplo, asumir que la diversidad de finalidades de una misma investigación científica puede abordarse desde las diferencias entre ambas perspectivas (Moyano, 1999). Así, para una problemática que implique leyes generalizables es más idónea una investigación de laboratorio orientada a 
lo cuantitativo, mientras que para un examen de sus consecuencias prácticas es más pertinente realizar una investigación-acción de tipo evidentemente cualitativo $^{8}$. Otra forma de lograr concesión aparece por el camino de las investigaciones mixtas, en ellas existen dos formas para aproximarse tanto cuantitativa como cualitativamente al fenómeno social. Por un lado, tendríamos el modelo mixto, que mezcla elementos cuantitativos (estadística, confirmación de hipótesis, predicción, explicación, etc.) y elementos cualitativos (descubrimiento, exploración, generación de teorías, etc.) en cada etapa, dependiendo de los requerimientos de la investigación (Johnson \& Onwuegbuzie, 2004). Pero por otro lado tendríamos el método mixto, referido a una investigación frente a un fenómeno social específico que se vale de cada una de las fases que componen ambas formas de investigación social ${ }^{9}$ (Johnson \& Onwuegbuzie, 2004). No obstante, y a pesar de estos esfuerzos el problema aún persiste, pues solo se está abarcando el ámbito metodológico y, como se afirmaba al comienzo, en el fondo de la discusión siguen estando principios filosóficos y epistemológicos arraigados. Este "fondo epistemológico-filosófico" de la discusión es todavía más fácil de visualizar si nos remitimos a la actualidad de la misma confrontación. Recientemente lo cuantitativo ha sido asociado al daño que existe en el planeta derivado de la explotación indiscriminada de recursos naturales, pues su forma de investigación tiende a la expansión de la cantidad de resultados y sus logros se vuelven un producto tecnológico. La consecuencia más importante de esta asociación es el aumento de la tensión entre los partidarios de uno y otro enfoque, sobre todo si consideramos que lo cualitativo se tiende a vincular al humanismo, el pluralismo y la calidad por sobre la cantidad (Neuenschwander, 2013). Como se puede constatar, todo esto es solo una versión más de la constante contienda entre quienes abogan por una preponderancia de lo material y fáctico como sentido de la realidad y los que abogan por lo conceptual, abstracto y espiritual como fundamento también de la misma realidad. Así, los alcances no solo son epistemológicos sino también filosóficos, pues la filosofía es una disciplina que replantea incesantemente los problemas, para aclarar con ellos el significado y la realidad de la vida humana (Abbagnano, 1994). En otros términos, la discusión entre cuantitativistas y cualitativistas implique cierta instalación y forma de dar sentido al mundo, lo que traslada la disputa al campo pleno de la filosofía.

Además de los problemas anteriores, también existe una dificultad para cohesionar las perspectivas metodológicas dentro del campo de la epistemología y en última instancia de la filosofía. Cuesta mucho que las propuestas alternativas al positivismo, tales como las criticas socioculturales provenientes del Programa Fuerte, Construccionismo Social, Teoría Crítica, Racionalismo, Feminismo, Complejidad, y las críticas epístemológicas propias de Marx, Kuhn, Popper, Fine, etc., constituyan un todo cohesionado en favor de ofrecer un único fundamento filosófico (Páramo \& Otálvaro, 2006). En efecto, lo único que se puede observar es más bien una pluralidad de críticas diversas a la evolución y construcción de teorías científicas (Páramo \& Otálvaro, 2006). Por otra parte, también se ha pretendido postular al pragmatismo como principio que oriente la cohesión entre el paradigma cualitativo y el paradigma cuantitativo (Johnson \& Onwuegbuzie, 2004), lo cual podría tener cierto alcance filosófico y con ello solucionar la discusión. Con el pragmatismo se busca darle una acentuación a los resultados de la investigación, se pretende una flexibilidad entre enfoques y una mezcla de los mismos en beneficio de los logros del trabajo científico. No obstante, el pragmatismo justamente por su dimensión práctica resulta más una orientación o ideología metodológica más que una propuesta filosófico-epistemológica con un planteamiento en torno al fundamento último de la actividad científica.

\section{LA HERMENÉUTICA GADAMERIANA Y LA CIRCULARIDAD DE LA COMPRENSIÓN EN VERDAD Y MÉTODO}

Si bien es cierto que la hermenéutica tiende a relacionarse con el cualitativismo y que las ideas del alemán Hans-Georg Gadamer se enmarcan dentro de una búsqueda de autodefinición de las ciencias del espíritu —o ciencias humanas-, también veremos cómo estas mismas ideas hacen que su planteamiento consiga tintes filosóficos universales. Un ejemplo de esta universalidad puede observarse en la deuda intelectual que el pensamiento apeliano mantiene con la propuesta gadameriana. En efecto, es tal el alcance de la hermenéutica filosófica de Gadamer que Apel la 
utiliza como referencia para desarrollar reflexiones en campos tan diversos como la ética, la semiótica y la filosofía del lenguaje (Scivoletto, 2018). De esta forma, veremos como la indagación en este sistema hermenéutico también se hace relevante a la hora de buscar un fundamento filosófico que establezca puentes de conexión entre las formas de investigación científica social.

Al hacer una síntesis general de la propuesta hermenéutica de Hans-Georg Gadamer, tenemos que se trata más bien de un llamado a poner atención en la historicidad del ser humano y del peso que esta tiene en su subjetividad, asumiendo que la interpretación (o el entender) solo se hace posible gracias a un acervo previo que tomaría forma de interrogante (Grondin, 1999). Además, dicha interrogante hace posible el inicio de un dialogo tanto a nivel interior como a nivel exterior (con otros sujetos), aportando a una interpelación frente a nosotros mismos y frente otros (Grondin, 1999). Este proceso siempre se da en relación a las cosas con el fin de darles sentido (entenderlas) dentro de un marco de expresión delimitado por el lenguaje y las cosas mismas. La Figura 2 deja más clara la estructura general de la hermenéutica gadameriana, ya que sus ideas aparecen interconectadas a través de la finitud subjetiva, la expectativa de sentido y la posibilidad de entender e interpretar.

\section{Figura 2.}

Estructura general de la hermenéutica gadameriana

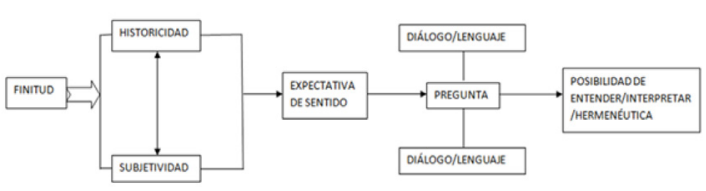

A partir de esta noción general en torno a la hermenéutica filosófica gadameriana se vislumbra el papel preponderante que juegan las preconcepciones a la hora de abordar la realidad, condición que le es propia a un ser consciente que se desenvuelve en una dimensión finita y por lo tanto histórica. Sin embargo, lo que más interesa de Gadamer en el contexto de la presente investigación es aquello que se logra captar a través del diálogo que inicia la pregunta forjada a partir de la propia historicidad, en otras palabras, la configuración circular que posee la comprensión o el entender. La importancia de esta idea como es de suponer, radica en la apertura de una oportunidad de conexión con la dinámica interna al funcionamiento del método científico (en su vertiente más positivista). Así pues, para entender a cabalidad la estructura circular de la comprensión y focalizarnos específicamente en la propuesta de este escrito, es necesario revisar directamente las ideas que Gadamer propone en la que, como se verá, es su obra fundamental Verdad y Método (Gadamer, 1998).

La estructura circular presente en la historia del planteamiento hermenéutico no es algo que esté como novedad en la filosofía de Gadamer. En efecto, hay vestigios durante la Antigüedad de la idea de que el todo se entienda por la parte y a su vez la parte por el todo. San Agustín en sus intentos de comprensión del escrito bíblico ${ }^{10}$ constituye una buena muestra de aquello (Calabrese, 2015). El autor cristiano destaca que los pasajes oscuros de la Biblia son comprensibles desde los pasajes claros, lo que a su vez ayuda a comprender la totalidad del escrito, totalidad que por lo demás sirve justamente para iluminar los pasajes oscuros que puedan seguir existiendo. A pesar de este carácter antiquísimo, en Verdad y Método se parte afirmando que la idea de la circularidad solo se hace explícita con Friedrich Schleiermacher durante la modernidad, éste sostiene que es necesario conocer la tradición y género en que se enmarca la obra para entender su particularidad. Así, esta comprensión de lo particular de la obra permitiría el conocimiento de su contexto creativo y anímico (Gadamer, 1998). Para Gadamer, las ideas del círculo hermenéutico que plantea Schleiermacher son un tanto erradas, pues si rememoramos los puntos tratados en el apartado anterior, hay una relevancia de la propia tradición a la hora de abordar un texto. Esto sin embargo no abre la posibilidad de justificar indistintamente las preconcepciones erradas que se tienen en torno al texto mismo. $\mathrm{La}$ idea presente en Verdad y Método es ir a las cosas (Gadamer, 1998). Así pues, el dirigirse a las cosas mismas, las que en mayor o menor grado están expresadas por medio del lenguaje de un texto, implica también un proyecto, un dirigirse hacia las cosas con una expectativa frente a ellas. No obstante, esta expectativa o proyecto puede verse modificada por el texto (la cosa) propiamente tal (Gadamer, 1998). En otras palabras, se enfrenta el texto a través de un movimiento de ida, el que está determinado por la necesidad de corroborar nuestra expectativa de sentido producto de la tradición (prejuicio). Así, el "proyecto" de acuerdo 
a Verdad y Método sería la esperanza de que el texto responda a la pregunta surgida desde nuestra propia expectativa de sentido. $\mathrm{Al}$ mismo tiempo, esto no implica en modo alguno un subjetivismo absoluto, pues es el texto (las cosas) el que corrige este proyecto, haciendo un movimiento de regreso hacia el sujeto que busca comprender. De este modo, podemos en efecto reconstruir la pregunta misma para la que el texto se estableció como respuesta, pues éste encamina y modifica nuestro proyecto-pregunta inicial. Además, lo anterior no evita que aparezcan nuevos proyectos o preguntas, puesto que la reconstrucción de la pregunta a la que el texto ofrece una respuesta está ella misma contextualizada dentro de un hacerse preguntas. Actividad con la que nosotros mismos intentamos buscar la respuesta a la interrogante que nos plantea la tradición o que generamos por medio de ella (Gadamer, 1997).

Gadamer añade otra característica al círculo hermenéutico ya descrito, que puede ayudarnos a entender mejor ese "remitirse a las cosas mismas" que sostiene siguiendo a Heidegger. Esta categoría, que denomina anticipo de la compleción, alude a ese supuesto previo bajo el cual asumimos que el texto que se nos presenta constituye una unidad de sentido acabada, lo que le da cierto estatus de verdad (Gadamer, 1998). No se trata de si el texto está completo o no, sino más bien de su manifestación con un sentido (o querer decir) completo. De este modo se remite a las cosas mismas o al texto mismo que las expresa, pues se anticipa un sentido pleno justamente a causa de que éste se nos manifiesta como una unidad de sentido ${ }^{11}$.

Sintetizando, la circularidad de la propuesta gadameriana parece evidente si se remite a la idea de que la posición previa del intérprete ilumina la comprensión del texto y las cosas que este expresa. Al mismo tiempo, es el texto (la cosa misma) quien corrige la apertura previa o la expectativa de sentido, la que se traduce en una pregunta a la que buscamos responder. Como veíamos previamente, todo esto tiene un ribete universal, pues para el filósofo alemán el lenguaje es el ambiente en el que nos desenvolvemos y el medio por el cual buscamos comprender las cosas (Cáceres, 2018). Así Gadamer se acerca a la idea heideggeriana de una hermenéutica con carácter ontológico, el proceso hermenéutico es algo que realizamos siempre (Gadamer, 1998), corresponde a nuestro estar conscientes en el mundo.

\section{HACIA UNA SÍNTESIS EPISTEMOLÓGICA DE LOS ENFOQUES PRESENTES EN LA INVESTIGACIÓN SOCIAL CIENTIIFICA}

Se sostenía anteriormente que es clara la necesidad de una propuesta filosófica y no solo epistemológica a la hora de solucionar el debate entre cuantitativistas y cualitativistas en la investigación social. Se requiere de una propuesta con alcance ontológico para lograr la anhelada unidad de enfoques. Pero además de esta profundidad filosófica, uno de los requisitos centrales es que la propuesta contenga dentro de sí elementos que conciernen tanto a uno como a otro enfoque. En este sentido la hermenéutica gadameriana se presenta como idónea para tal fin. Esto se debe principalmente a tres motivos. Primero, encontramos que Gadamer le da un estatus universal al lenguaje y a la interpretación (Gadamer, 1998), lo que otorga a su planteamiento un peso epistemológico-filosófico considerable a la hora de pensar en un principio para la realidad, o al menos, para la comprensión de ésta. En segundo lugar, la importancia que le da a la interpretación y al lenguaje (Gadamer, 1998) hace que las ideas del filósofo alemán se familiaricen con la búsqueda de conceptualización propia de las investigaciones de carácter cualitativo. Finalmente, la circularidad de su propuesta hermenéutica, y más específicamente, de la actividad de comprensión ejercida por la conciencia humana, la acercan a la forma tradicional de concebir el método científico presente en el positivismo. Para entender este último punto es necesario detenerse y ofrecer una explicación más detallada en favor de comprender su real alcance. Decíamos que por su dinámica interna le era inherente al método científico cierta circularidad, la que se refleja en el movimiento de ida y de vuelta -implicando deducción e inducción- que la ciencia hace al ir desde la teoría a los datos y desde los datos a la teoría, en un intento de corroborar la hipótesis planteada y aportar al acervo teórico existente. Así pues, en sintonía con este "sistema de funcionamiento" del método científico, la hermenéutica de Gadamer plantea algo similar, debido a que destaca un movimiento de ida que implica ir desde el sujeto (tradición) hacia el texto (o las cosas), y también un movimiento de regreso que va desde el texto al sujeto. En efecto, el sujeto gracias a poseer un conjunto de conocimientos previos, o tradición, posee también una expectativa de sentido 
que debe comprobar frente al texto formado por el lenguaje y que expresa las cosas, lo cual acerca a la hermenéutica de Gadamer a la formulación de preguntas y a la búsqueda de corroboración de una hipótesis. Recordemos que estos últimos son los ejes epistemológicos claves que se utilizan para guiar una investigación científica (Tello, 2011). Además, tenemos que es el texto mismo el que corrige o responde al intérprete en su corroboración de la expectativa de sentido previa. Así, una confirmación correcta de la expectativa de sentido permite llegar al "querer decir del texto", al igual que la ratificación de la hipótesis permite establecer un principio que modifica o mantiene la teoría científica imperante. La Figura 3 presenta ambos tipos de movimientos circulares en paralelo, lo que ayuda a iluminar el vínculo existente entre cualitativismo y cuantitativismo:

Figura 3.

Circularidad metódico-científica y hermenéutica
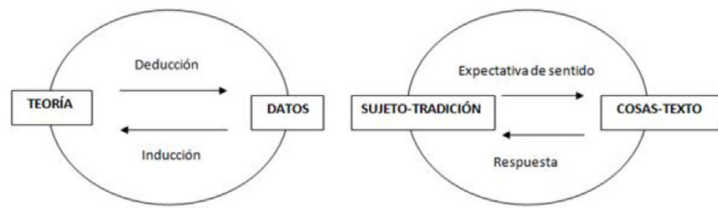

Se puede agregar a lo ya dicho, que la universalidad de las ideas gadamerianas se ve confirmada también a través de su circularidad, pues inusitadamente esta permite un punto de comunicación o diálogo concreto entre una posición en principio cualitativa y otra radicalmente opuesta como lo es el positivismo cuantitativista. Se saca así a la filosofía de su universalidad abstracta para llevarla a una universalidad concreta y ejemplificable. Por otra parte, se podría objetar también que Gadamer hace un giro hacia el sujeto, algo que siempre se le critica las filosofías de tipo hermenéutico. Sin embargo, también Gadamer es enfático en ese "ir a las cosas mismas", en ese dejar que el objeto hable también, lo que en clave positivista puede entenderse como el dejar hablar a la naturaleza por medio de los datos obtenidos en el experimento.

\section{A MODO DE SÍNTESIS Y CONCLUSIÓN}

A lo largo del escrito se expuso como el método científico tradicional, mucho más cercano a las ciencias naturales, es difícil de ser aplicado en ciencias sociales. El origen de este inconveniente radica en que los problemas socioculturales muchas veces tienen a su base elementos que se escapan a la experimentación, cuantificación y observación directa. Esto lleva al surgimiento de posturas que acentúan la cualidad y la conceptualización en el campo de la investigación social más allá de la experimentación. Aparece así la confrontación entre cuantitativistas y cualitativistas, donde los primeros creen que el método imperante en las ciencias naturales es aplicable completamente a la sociedad, mientras que los segundos tratan de interpretar la cultura atribuyendo conceptos y estructuras a los fenómenos sociales. Como se constató, la oposición entre uno y otro enfoque metodológico está determinada por factores epistemológicos y filosóficos, pues dependen en último término de una manera particular de concebir la realidad en su totalidad. Así, por un lado, los cuantitativistas dan cuenta de los problemas sociales a través de las cosas y la naturaleza (acentuando la experimentación, la clasificación y la observación), mientras que los cualitativistas lo hacen por medio de las ideas y los conceptos (acentuando la observación y la crítica). Toda esta situación parece llevar a la urgencia por un pensamiento filosófico que traiga la conciliación en medio de la confrontación entre ambos enfoques. En este marco, la reflexión del alemán Hans-Georg Gadamer se muestra apropiada para establecer un lugar neutral de diálogo en la discusión, puesto que su propuesta ofrece una fundamentación del lenguaje y la comprensión como contexto universal, dando paso a una instalación determinada en el mundo. Además de esto, Gadamer conecta con el enfoque cualitativo en su tendencia interpretativista, debido a la acentuación que pone en la comprensión como algo inevitable en el sujeto, pero también conecta con el enfoque cuantitativo de tendencia más positivista, a partir de la acepción más tradicional (y circular) del método científico que lo caracteriza. 


\section{Referencias}

Abbagnano, N. (1994). Historia de la filosofía. Barcelona: Hora.

Agustín, S. (2001). Confesiones. Madrid: Biblioteca de autores cristianos.

Bunge, M. \& Ardila, R. (1988). Filosofía de la psicología. Barcelona: Ariel.

Cáceres, A. (2018). Verdad y Método. El lenguaje como experiencia humana en la conciencia de la historia y en el arte poético: Hans Georg Gadamer. Pensamientos, 74(282), 963-977. Recuperado de https://revistas.comillas.edu/index.php/pensamiento/article/ download/7198/pdf

Calabrese, C. (2015). Los supuestos hermenéuticos de Agustín de Hipona. Desentrañar la palabra y transmitir su misterio. Espíritu, 64(150), 227-243. Recuperado de https://dialnet. unirioja.es/descarga/articulo/5244646.pdf

De Souza Nogueira, P. (2013). Hermenêutica da Recepção: Textos bíblicos nas fronteiras da cultura e no longo tempo. Estudos de Religião, 26(42), 1-7. Recuperado de https://www. metodista.br/revistas/revistas-metodista/index.php/ER/article/ viewFile/3095/3086

Feyerabend, P. (1985). Tratado contra el método. Esquema de una teoría anarquista del conocimiento. Madrid: Tecnos.

Gadamer, H. (1997). Verdad y Método, tomo I (7ª ed.). Salamanca: Sígueme.

Gadamer, H. (1998). Verdad y Método, tomo II (7 $\left.{ }^{\mathrm{a}} \mathrm{ed}.\right)$. Salamanca: Sígueme.

Grondin, J. (1999). Introducción a la hermenéutica filosófica. Barcelona: Herder.

Guba, E. (1990). The alternative paradigm dialog. En E. Guba, (Ed.), The paradigm dialog (pp. 17-27). Newbury Park, CA: Sage.

Johnson, R. \& Onwuegbuzie, A. (2004). Mixes methods research. A research paradigm whose time has come. Educational Researcher, 33(7), 4-26. doi: 10.3102/0013189X033007014

Kuhn, T. (2013). La estructura de las revoluciones científicas. México D. F.: Fondo de cultura económica.
Landeros-Olvera, E., Salazar-González, B., \& CruzQuevedo, E. (2009). La influencia del positivismo en la investigación y práctica de enfermería. Index de Enfermería, 18(4), 263-266. Recuperado de http://scielo.isciii.es/scielo. php?script=sci_arttext\&pid=S1132-12962009000400011

León, O. \& Montero, I. (2003). Métodos de investigación en psicología y educación. Madrid: McGraw Hill.

Moyano, E. (1999). Hacia la integración de métodos cualitativos y cuantitativos en la investigación social. Revista de la facultad de humanidades, 4, 54-86.

Neuenschwander, E. (2013). Qualitas and Quantitas. Two ways of thinking in Science. Qual. Quant., 47, 2597-2615. doi: 10.1007/ s11135-012-9674-7

Páramo, P. \& Otálvaro, G. (2006). Investigación Alternativa: Por una distinción entre posturas epistemológicas y no entre métodos. Cinta de Moebio, 25, 1-7. Recuperado de http://www. redalyc.org/articulo.oa?id=10102501

Pereira, H. \& Martins, J. (2013). O Cientificismocomo Obstáculo à Compreensão ÉticoPolítica da Psicologia [Sección especial]. Psicologia: ciência e profissão, 33, 42-53. Recuperado de http://www.scielo.br/scielo.php?script=sci_abstract\&pi$\mathrm{d}=$ S1414-98932013000500006\&lng=en\&nrm=iso\&tlng=es

Scivoletto, G. (2018). Apel y la hermenéutica filosófica de Gadamer. ARETÉ. Revista de filosofía, 30(2), 333-355. dio: 10.18800/arete.201802.007

Scribano, A. (2000). Reflexiones epistemológicas sobre la investigación cualitativa en ciencias sociales. Cinta de Moebio, 8, 128-136. Recuperado de http://www.redalyc.org/ pdf/101/10100802.pdf

Sierra, R. (1987). Técnicas de investigación social. Teoría y ejercicios. Madrid: Parainfo.

Tello, C. (2011). El objeto de estudio en ciencias sociales: entre la pregunta y la hipótesis. Cinta de Moebio, 42, 225-242. Recuperado de http://www.redalyc.org/articulo.oa?id=10121113001 


\section{Notas al final}

1 Esto se puede ejemplificar si tomamos el caso específico de la psicología social. Para Pereira dos Santos y Martins (2013), la psicología social le atribuye a la obra de Thomas Kuhn: Estructura de las revoluciones científicas, un carácter más prescriptivo que descriptivo. En efecto, el texto de Kuhn pretendía describir cómo se producen los cambios en las ciencias poniendo especial atención en la transformación de sus paradigmas (métodos, modelos, técnicas y teorías que la orientan) y su constitución en una ciencia normal, en la que hay estabilidad o firmeza en la obtención de resultados. Justamente, la física se muestra como una ciencia normal por antonomasia y desde la psicología, que aspira a convertirse en ciencia normal, se sigue su modelo o paradigma (Pereira dos Santos \& Martins, 2013).

2 Es decir, a partir de la forma que toma aproximadamente desde el siglo XVII.

3 Bunge y Ardila (1988) distinguen más pasos dentro del método científico, los cuales, sin embargo, pueden agruparse dentro de las tres fases que distingue Sierra (1987). Las operaciones que proponen Bunge y Ardila son las siguientes: a) Identificar un problema, b) Formular el problema con claridad, c) Buscar información e instrumentos pertinentes, d) Tratar de resolver el problema con los medios recopilados, e) Inventar nuevas ideas, f) Obtener una solución, g) Extraer consecuencias a partir de la solución, h) Controlar la solución propuesta (experimentación, i) Corregir la solución si es defectuosa, j) Examinar el impacto de la solución sobre el cuerpo teórico establecido.

4 Es común encontrar en Sierra (1987) la utilización sinonímica entre las nociones de verificación de la realidad, confirmación de la hipótesis y corroboración a partir de los hechos. Sin embargo, la primera resulta controversial debido a la carga interpretativa que tiene el conocimiento, característica que se profundizará en este escrito a partir de las ideas que Gadamer plantea a través de su hermenéutica filosófica. Por este motivo se ha optado por utilizar solamente las otras dos conceptualizaciones a lo largo de la exposición.

5 Las metodologías más usadas a nivel cualitativo son tres. La etnografía, que busca describir e interpretar el comportamiento de un grupo que comparte una cultura en función de un significado simbólico (León \& Montero, 2003). El estudio de casos, que busca interpretaciones y generalizaciones a partir de un caso único (León \& Montero, 2003). Y finalmente la investigación-acción, que se caracteriza por interpretar una realidad social teniendo como meta su modificación y mejoramiento.

6 A este respecto, no es irrelevante recordar que el enfoque cuantitativo recibe este nombre justamente por su tendencia a cuantificar o asignar números a variables (medidas en magnitudes), las que se busca relacionar por medio de la observación y la experimentación.

7 El Positivismo, cuya raíz etimológica es la palabra latina positum, que a su vez alude a "lo que ocupa una posición en el espacio", se refiere a una perspectiva filosófico-científica impulsada mayormente por el francés Augusto Comte en el siglo XIX. Ésta sostiene que solo son relevantes para el conocimiento las leyes que se pueden obtener a partir de la experimentación y los fenómenos observables. El positivismo abandona así toda posibilidad de obtener una verdad de tipo metafísico y da a entender que la ciencia experimental representa el desenlace definitivo de la historia del conocimiento humano (Landeros-Olivera, Salazar-González \& Cruz-Quevedo, 2009).

8 Para obtener una visión panorámica más amplia de las diversas problemáticas científicas y las múltiples formas de investigación que se pueden asociar, se recomienda revisar el cuadro que aparece en: Moyano, E. (1999). Hacia la integración de métodos cualitativos y cuantitativos en la investigación social. Revista de la facultad de humanidades, 4, 54-86.

9 Si bien las fases de investigación dentro de una perspectiva cuantitativa se enmarcan dentro del proceso circular inherente al método científico, cuando hablamos solamente de la investigación cualitativa estas fases son un poco más difusas. De hecho, León y Montero (2003) prefieren hablar de técnicas o metodologías de investigación cualitativas en lugar de un método preciso, destacando que tanto la etnografía, la investigación-acción y el estudio de casos deben ser flexibles en sus etapas, pues muchas veces los criterios de investigación vienen dados por la interpretación que se va construyendo constantemente durante el trabajo científico aplicado a una realidad sociocultural.

$10 \quad$ Sobre todo, en los últimos tres libros de las Confesiones.

11 Gadamer grafica esto a través del ejemplo de la recepción de una carta o la revisión de las noticias en el periódico (Gadamer, 1998). En ambos casos el lector de la carta o el periódico asume la posición del remitente o el corresponsal que le está informando, pues reconoce que en el texto mismo se encuentra (o manifiesta) una unidad de sentido. 